\section{$(x)$ \\ HORIZONTES}

www.revistahorizontes.org
Horizontes. Revista de Investigación en Ciencias de la Educación Https://doi.org/10.33996/revistahorizontes.v3i11.79

julio - septiembre 2019

Volumen 3 / No. 11

pp. 188 - 201

\title{
Estrategias metodológicas educativas en el fortalecimiento de los procesos de la autoevaluación de estudiantes de cuarto de secundaria en Bolivia
}

\author{
Educational methodological strategies in strengthening the processes of \\ self-assessment of secondary school students in Bolivia
}

Franz Higueras Vargas

franzhiguerasvargas@gmail.com

Universidad Mayor Real y Pontificia San Francisco Xavier de Chuquisaca, Bolivia

\section{RESUMEN}

En el presente artículo está relacionado con la relevancia actual desde el ámbito educativo, social y cultural; dedicado a la falta de innovación en la práctica Educativa de la autoevaluación. Su objetivo fue determinar el tipo de estrategias metodológicas educativas de la Autoevaluación en el Campo Vida Tierra y Territorio para el fortalecimiento de habilidades y potencialidades de autorreflexión, autocrítica, conciencia social y actitud personal de las y los estudiantes de secundaria. El proceso metodológico estuvo enmarcado bajo el paradigma cualitativo interpretativo $y$ explicativo de tipo correlacional. El objeto del diagnóstico de estudio, que se realizó en la institución Educativa, por medio de instrumentos técnicas que permitieron la obtención, de información acerca de las Estrategias Metodológicas Educativas para fortalecimiento de los procesos de la Autoevaluación, posteriormente mediante la tabulación de los resultados se analizaron e interpretaron, resultando un modelo teórico diseñado para contrarrestar la problemática identificada, también se planteó los fundamentos teóricos, enfoques, paradigmas y la descripción de su estructura en base de un plan de cada estrategia. Al finalizar se realizó la validación Empírica, el cual fue llevado a la práctica las "Estrategias Metodológicas educativas", de acuerdo a los resultados obtenidos de preguntas de test, se pudo evidenciar que las actividades son válidas para su aplicación en la Unidad Educativa Libertad de las Américas "A".

Palabras clave: Estrategias metodológicas educativas; autoevaluación

\begin{abstract}
In this article it is related to the current relevance from the educational, social and cultural field; dedicated to the lack of innovation in the educational practice of selfevaluation. Its objective was to determine the type of educational methodological strategies of the Self-Assessment in the Land Life and Territory Field for the strengthening of skills and potential of self-reflection, self-criticism, social awareness and personal attitude of secondary school students. The methodological process was framed under the qualitative interpretive and explanatory paradigm of a correlational type. The purpose of the study diagnosis, which was carried out in the Educational Institution, by means of technical instruments that allowed the obtaining, of information about the Educational Methodological Strategies for strengthening the Self-Assessment processes, later by tabulating the results analyzed and interpreted, resulting in a theoretical model designed to counteract the identified problem, the theoretical foundations, approaches, paradigms and the description of its structure based on a plan of each strategy were also raised. At the end, the Empirical validation was carried out, which was carried out the "Educational Methodological Strategies", according to the results obtained from test questions, it was possible to show that the activities are valid for their application in the Freedom of Education Educational Unit the Americas "A".
\end{abstract}

Key words: Educational methodological strategies; self-appraisal 
INTRODUCCIÓN

En la sociedad actual específicamente en el campo de la educación el proceso de enseñanza y aprendizaje y la evaluación educativa permanecen estrechamente relacionadas entre sí, debido a su tiempo y dinámica continua. Por lo que es necesario en la formación en valores pedagógicos a la práctica evaluativa, la cual debe diseñarse para el estudiante, enriqueciendo su proceso de formación y respondiendo a su necesidad específica de conocimientos.

La transformación Educativa que hoy en día se quiere establecer en Bolivia según La ley 070 "Avelino Siñani Elizardo Pérez" fomentada desde el Sistema Educativo Plurinacional (SEP) en la Educación Regular en el nivel secundario, creando y consolidando espacios educativos en los cuales se utilicen adecuadamente la Autoevaluación ("proceso formativo en el que el estudiante es un agente activo de su propio aprendizaje").

Debido a que la sociedad actual se encuentra invadida por la falta de ética y valores en los adolescentes una preocupación que constantemente se ha convertido en el centro de investigaciones, reflexiones y discusiones, al ser relevante para la enseñanza y el aprendizaje. Mediante la ley 070 "Avelino Siñani-Elizardo Pérez". Por lo que en la educación se trata de incorporar la autoevaluación para que sean empleadas y se conviertan en el medio por cual se consigan óptimos resultados en el rendimiento académico, pero ante la mala utilización y caos que genera, se debe orientar y capacitar tanto a los profesores y los estudiantes acerca del buen uso y manejo para formar generaciones productivas que busquen no solo el bienestar individual sino el bienestar común orientado hacia el Vivir Bien entre las personas (Huanacuni, 2010). Necesariamente gran parte de los procesos educativos tradicionales deben ser reestructurados, modificados y adaptados a las nuevas generaciones acorde a su realidad, tomando en cuenta las nuevas formas innovadoras de proyectar la educación, creando un clima agradable en el aula plena en valores sociocomunitarios vinculados a la consolidación de saberes y conocimientos propios.

Las relaciones interpersonales son el pilar fundamental para concretar una nueva educación donde los espacios educativos se conviertan en espacios de dialogo, de intercambio de ideas, puntos de vista $\mathrm{y}$ experiencias, por medio de prácticas educativas que fomenten a la participación individual, colectiva. Entre el profesor y los estudiantes, donde las experiencias educativas beneficien a la comunidad educativa de manera productiva.

Hoy en día hablar de la Autoevaluación de los procesos de Enseñanza y Aprendizaje suena algo distante y difícil de poder lograr para muchos de los profesores, por lo cual, aún existen estudiantes que no son conscientes de lo que significa autoevaluarse y que se puedan hacerse una autocrítica de su propia formación, actualmente con la ley 070 las nuevas tendencias $\mathrm{y}$ exigencias del bachiller técnico humanístico, que estos una formación integral y holístico desarrollando su personalidad en sus cuatro dimensiones (Ser, Saber, Hacer y Decidir). Como también la formación sea útil para afrontar los estudios en la universidad y poder lograr una formación profesional adecuada y de calidad para posteriormente ayudar a las nuevas generaciones.

Este artículo estará dedicado al proceso de formación mediante construcción de estrategias metodológicas Educativas que contribuyan al fortalecimiento de los procesos de la Autoevaluación de estudiantes de Secundaria. La investigación se realizó con estudiantes de ambos sexos del Cuarto años 
de Secundaria, con edades comprendidas entre 15 y 16 años y los profesores pertenecientes al Campo Vida Tierra y Territorio, en la "Unidad Educativa Libertad de las Américas. La investigación trata de determinar el tipo de estrategias Metodológicas Educativas utilizadas en los Procesos de la Autoevaluación para los estudiantes en el Campo Vida Tierra y Territorio del Nivel Secundario de la Unidad Educativa Libertad de las Américas " $A$ " de acuerdo a la ley 0.70 Avelino Siñani y Elizardo Pérez. (Ministerio de Educación 2014).

Socialmente fue pertinente estudiar las diferentes Estrategias utilizadas actualmente por los profesores en el Sistema Educativo Plurinacional ya que estos deben ser diseñados adecuadamente para que las y los estudiantes puedan realizar una Autoevaluación apropiada durante la finalización del bimestre sin reducir ni exceder la nota que les corresponde, ya que hoy en día las calificaciones realizadas por los estudiantes en la autoevaluación no son aceptables por sus propios profesores Además la Autoevaluación se encuentra sustentado dentro de la ley 070 "Avelino Siñani Elizardo Pérez" que hoy en día se encuentra vigente, como también se pudo ver que es necesario que los estudiantes se valoren cualitativa y cuantitativamente por sí mismos y ellos puedan hacer una autocrítica de su propia formación debido a que la sociedad actual que refleja la falta de valores y conciencia.

La investigación de este tema se consideró novedosa dentro del contexto donde se desarrollaron los procesos de la Autoevaluación, en gran medida el resultado de la autovaloración de los estudiantes genera la autocrítica de su propia formación. A la vez se reflexiona sobre el comportamiento, la forma de ser y actuar, ayudándoles a crecer en su dignidad como persona en la sociedad.
También es novedosa porque busca determinar el tipo de Estrategias Metodológicas Educativas utilizadas por el profesores en la Autoevaluación para los estudiantes al finalizar el bimestre, priorizando la adecuada aplicación de la ley 070 "Avelino Siñani Elizardo Pérez" dentro de la formación integral y holística establecida.

Actualmente se presenta en la sociedad transformaciones sociales y culturales, que inciden y exige una lectura desde la educación, parte fundamental en la construcción de la sociedad; articulada por una serie de sistemas que moldean al individuo de acuerdo a las necesidades y proyecciones, quien en convivencia adquiere herramientas que le permiten hacer su propia lectura de los códigos y signos que la sociedad le emite, para entender la realidad en la que está inmerso.

La educación, entendida como el proceso por el cual el hombre puede alcanzar su pleno desarrollo en los ámbitos físico, biológico, emocional, intelectual y espiritual al irse socializando, lo que incluye el conocimiento de los valores en los que se sustenta la vida diaria y que se traducen en actitudes, conductas y comportamientos que regulan cualquier actividad personal, familiar o social

\section{MÉTODO}

Fue un estudio factible ya que se pudo interactuar directamente con la realidad, además de acceder a diferentes fuentes de información bibliográficas, de archivos en internet, se pudo recabar datos, tanto de los profesores como de los estudiantes para realizar un análisis de la situación problemática y a partir de eso establecer alternativas de solución, no genero grandes gastos económicos por lo que el estudio resulto accesible.

En cuanto a la metodología en principio fue exploratorio sobre todo para detectar las 
situaciones problemáticas y establecer una única problemática de estudio y explorar alternativas solubles. También se tomó hacia un tipo de estudio descriptivo que sirvió para analizar cómo se manifiesta el fenómeno educativo tradicional en nuestros días, $\mathrm{y}$ cuáles deben ser los procesos educativos actuales a través de este estudio poder establecer alternativas que coadyuven la labor de los profesores/as y el buen desempeño de los estudiantes. Finalmente el estudio investigativo fue de tipo correlacional tiene, en alguna medida, un valor explicativo, aunque parcial, ya que el hecho de saber que dos conceptos o variables se relacionan aportan cierta información explicativa en este caso el diseño de las estrategias metodológicas en la autoevaluación para el fortalecimiento de las habilidades $y$ potencialidades de autorreflexión, autocrítica, conciencia social y actitud personal de las y los estudiantes en el aula, en el campo vida tierra y territorio.

El proceso investigativo en un principio se basó en la utilización de métodos teóricos y empíricos para determinar el diseño teórico y metodológico se utilizó instrumentos de la recolección de información y datos, que estuvo influida por experiencias y prioridades de los participantes, se hizo entrevistas, cuestionarios y observaciones no estructuradas de esta manera poder determinar la situación real de la problemática para plantear una situación ideal del fenómeno estudiado, estableciendo objetivos de manera pertinente, posteriormente con la construcción de modelo teórico que permitió realizar la validación empírica mediante la práctica en los estudiantes de cuarto de secundaria "A" de la Unidad Educativa Libertada de las Américas " $A$ ", para valorar el planteamiento de variables del estudio investigativo realizado.
RESULTADOS

La modelación teórica permitió establecer una relación directa entre el principal elemento de investigación presente en la realidad y la alternativa ideal para poder solucionarla, la misma está fundamentada en teorías, enfoques y posturas que se adopta de manera sistemática, se identificó los principales componentes y su estructura funcional para contrarrestar la problemática de manera eficaz consolidando una alternativa pertinente en la realidad de la comunidad educativa, en este propósito inicialmente se tomó en cuenta las conclusiones del diagnóstico realizado donde constata la falta de actividades y procedimientos educativos, interactivos, dinámicos y participativos ya que no existe la innovación de los procesos de enseñanza y aprendizaje en cuarto de secundaria "A" en el Área campo Vida Tierra y Territorio, donde se ve la necesidad de vincular el uso de estrategias metodológicas educativas que aportan significativamente a la formación integral de los estudiantes en el aula.

También es importante señalar que para la elaboración del modelo. No basto solo los resultados obtenidos del diagnóstico. Sino fue de vital importancia el conocimiento teórico del objeto de estudio el cual facilitó, el análisis y caracterización de tema.

\section{Estado real del objeto de estudio}

El estado real del objeto de estudio permitió modelar la información de los resultados exactos de los datos obtenidos en el diagnóstico, análisis de documentos, y la aplicación de instrumentos (cuestionario, guía de entrevista), también se toma en cuenta las pre-conclusiones del diagnóstico, de cada una de las preguntas el análisis e interpretaciones de los resultados correspondientes, y posteriormente las conclusiones generales del diagnóstico del objeto de estudio. 
Los estudiantes no cuentan con los elementos pertinentes para realizar una autoevaluación que aporte a su proceso de formación, siendo esto reflejo la falta de apropiación por parte del profesor la autoevaluación del estudiante; quien debe brindarle elementos a este para que la involucren en su contexto académico y como parte de su cotidianidad.

Para algunos profesores la autoevaluación es un momento enigmático ya que es la oportunidad de contrarrestar las notas bajas que han obtenido durante uno de los cuatro bimestres, para otros estudiantes es un momento de confusión al no saber cómo autoevaluarse de acuerdo a los criterios particulares del docente; acudiendo a los compañeros más cercanos para que en tono bajo le ayude a autoevaluarse y le digan una nota, y no arriesgarse a que el docente le manifieste gestualmente o verbalmente su inconformidad con la valoración.

\section{Estado ideal del objeto de estudio}

Reflejo, lo que se quiere alcanzar con lo que se propusieron a realizar o desarrollar con este estudio a partir de la realidad. Lo cual permitió transformar la realidad, para prevenir cualquier problema que se presente hacia el futuro, también la estructura de los componentes y sus relaciones permitirán tener una idea clara del objeto de estudio, es decir para mejorar el hábito de la lectura comprensiva en los estudiantes del nivel secundario.

La autoevaluación debe contribuir al estudiante durante su formación técnica y humanística, contribuyendo en el proyecto de vida, se debe re-direccionar los procesos de enseñanza y aprendizaje hacia un proceso autoconsciente de asimilación de saberes y conocimientos pertinentes, también deben fortalecer en el estudiante la parte axiológica y sus expectativas, ya que se va exigir para ser mejor, lo cual se debe ver reflejado en su futuro; las aspiraciones de estos estudiantes en la mayoría de los casos son limitadas, debido a las condiciones en que viven, sin embargo la autoevaluación debe contribuir para que crea en sí mismo y pueda colocarse retos a nivel académico y personales que con disciplina y auto exigencia puedan alcanzar.

Los profesores deben generar espacios de encuentro, análisis, reflexión, autocritica, autoconciencia entre los estudiantes, mediante experiencias significativas, donde la autoevaluación exija confianza emocional y socio-afectiva en el aula y esta haya transformado de manera paulatina $\mathrm{y} / \mathrm{o}$ trascendental los procesos de aprendizaje, ya que esto contribuye de manera significativa.

\section{Enfoque del modelo teórico}

Se asume el enfoque humanista, según el autor (Palacios 1998. p. 9) este enfoque aparte de realizar la evaluación de los procesos de enseñanza y aprendizaje usando diferentes criterios, propone la autoevaluación como una opción válida y complementaria donde los estudiantes con sus propios criterios están en una posición más adecuada para determinar en qué condiciones de aprendizaje y crecimiento se encuentran después de haber finalizado un curso o una actividad de aprendizaje.

En el enfoque humanista menciona Hernández (2010, Pp. 113 y 114) que es difícil realizar la evaluación usando criterios externos, por ello se propone la autoevaluación como una opción válida. Son los alumnos quienes, con base en sus propios criterios, están en una posición más adecuada para determinan en qué condiciones de aprendizaje y crecimiento se encuentran después de haber finalizado un curso o una actividad de aprendizaje. 


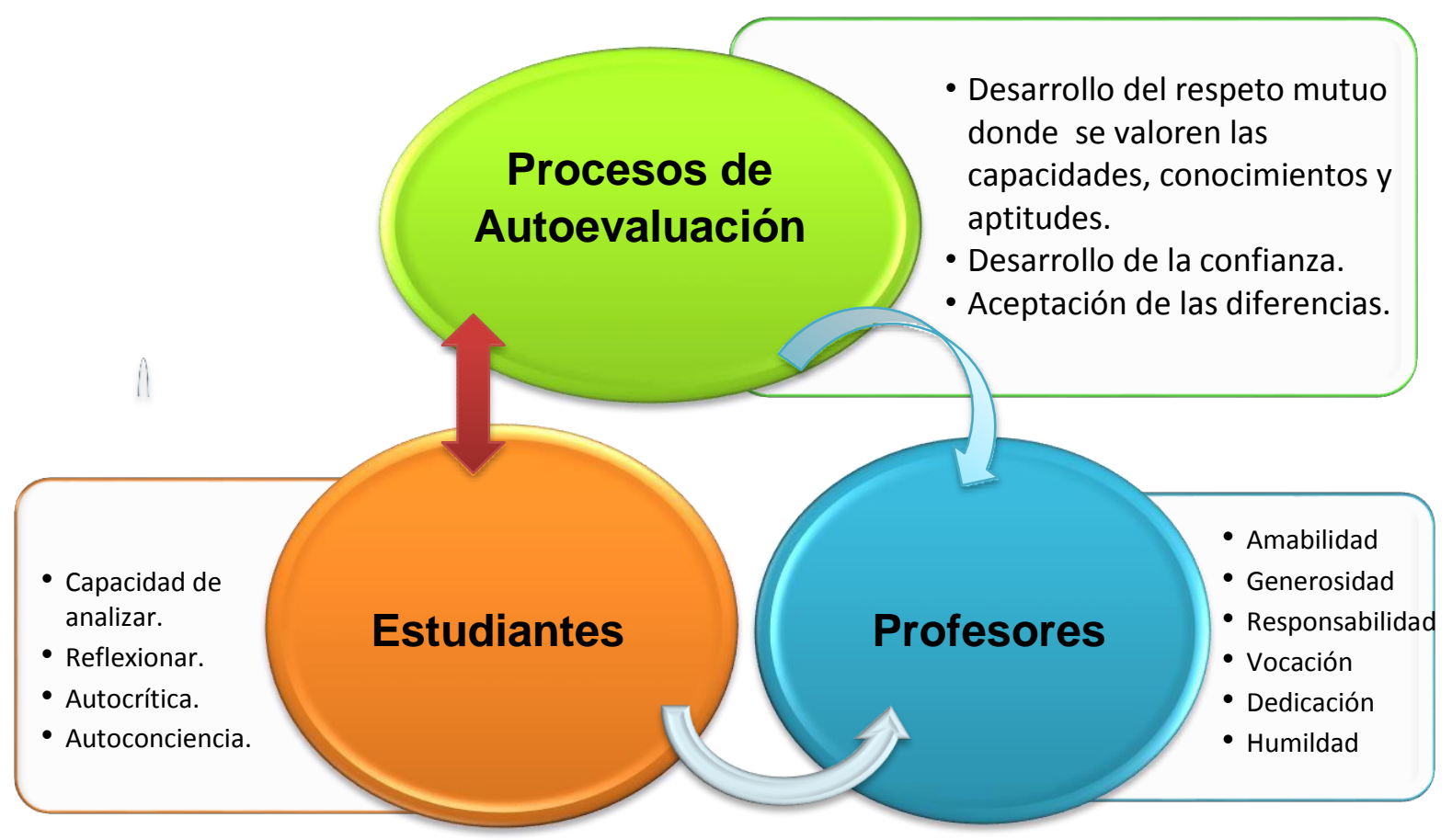

Figura 1. Componentes del modelo teórico basado en las metodologías propuestas en este estudio

\section{Componentes}

Los componentes identificados de acuerdo al marco teórico asumido y al enfoque expuesto. Son tres los cuales tienen una relevancia e importancia en su funcionalidad y desenvolvimiento dentro de los procesos Autoevaluativos de la enseñanza y aprendizaje de manera más efectiva, a partir de las cuales tienen una relación e interacciones de manera recíproca dentro del aula o fuera del aula.

\section{Características de los componentes Estudiantes}

Los estudiantes de cuarto de secundaria de la unidad educativa Libertad de las Américas " $\mathrm{A}$ " son un total de 31 estudiantes en donde el $61 \%$ son de sexo femenino y el resto el 39\% de sexo masculino. Como también la mayoría de los estudiantes vive al entorno y zonas cercanas a la unidad educativa, el $35 \%$ de ellos tienen la edad comprendida entre los 15 años, y los demás son compartidos entre los 16 y 17 . Dicha edad corresponde al nivel educativo al cual pertenecen ambos géneros como también ambos tienen las condiciones y características físicas e intelectuales para desarrollar los procesos formativos establecidos en el aula.

Roles del estudiante que debe cumplir durante su desarrollo y desenvolvimiento en el aula. Un estudiante hace una evaluación sobre sí misma, sobre una capacidad propia que desee valorar. Hacer sobre sí mismo sus fortalezas y debilidades, revisar sus procesos y su funcionamiento en general. Con habilidades y potencialidades de autorreflexión, autocrítica, conciencia social y actitud personal desde sus procesos de autovaloración, ser participativos, activos, solidarios, responsables, puntuales $\mathrm{y}$ respetuosos durante y después de todo su desarrollo formativo así para poder contribuir en un futuro a la sociedad. 


\section{Profesores}

Campo vida tierra y territorio esta concepción profundiza la formación integral y consiente de las y los estudiantes, valorando nuestros recursos naturales del contexto territorial donde se desarrollan las potencialidades $\mathrm{y}$ las vocaciones socio productivas con sustentabilidad

Consolidamos la formación técnico humanístico integral y holístico en reciprocidad, complementariedad y armonía con la madre tierra y el cosmos, mediante el estudio y análisis de los saberes $\mathrm{y}$ conocimientos científicos, técnico tecnológicos, propios y de otras culturas, aplicando practicas investigativas, innovadoras y creativas de acuerdo a las vocaciones y potencialidades productivas de las regiones, para transformar la comunidad.

Un profesor debe poseer un conjunto de competencias donde debe participar activamente en el desarrollo de las potencialidades individuales sociales respetar las diversas culturas de cada una de sus estudiantes y para ello debe de ser facilitador, mediador y una guía para lograr un mejor aprendizaje significativo.

Un profesor debe concebir competencias primordiales a saber

- Vocación: Sentir la profesión desde lo más profundo

- Dedicación: Emplear todo el tiempo disponible

- Amabilidad: Sin caer en el colegimos

- Generosidad: Volcar todos sus conocimientos en los estudiantes

- Responsabilidad

- Empatía: Saber en el lugar del alumno

- Humildad: No creerse que esté por encima de los demás

- Entusiasmó: Saber trasmitir energía vital

- Paciencia: No todos cogen las ideas al vuelo
- Saber otorgar protagonismo a los estudiantes

- Capacidad de una escucha activa

- Ser muy gráfico: una imagen vale que mil palabras

- Ser realista

Un buen profesor es aquel que es capaz de tener la curiosidad de aprender todos los días algo nuevo incluso de sus estudiantes

\section{Procesos de autoevaluación}

La autoevaluación es un proceso de reflexión consciente que realizan las y los estudiantes sobre la responsabilidad que tienen respecto de su formación y desarrollo de sus capacidades, cualidades y potencialidades, por lo que tiene también carácter formativo en tanto promueve la práctica de la honestidad y la responsabilidad con uno mismo y con la comunidad. Promueve, además, el desarrollo responsable de sus dimensiones.

En esta perspectiva, las niñas y niños, desde los primeros años de escolaridad, van cultivando y desarrollando el sentido de la honestidad al asumir la responsabilidad de valorarse a sí mismos bajo la guía $\mathrm{y}$ orientación de las profesores/as. Al saber que las calificaciones que obtenga la o el estudiante como resultado de las autoevaluaciones dependen de los registros que realice en el transcurso del bimestre respecto de la manifestación de sus capacidades, cualidades y potencialidades a partir de las cuatro dimensiones, cada estudiante se esforzará por poner en práctica, y también desarrollar, cada una de sus dimensiones. Por ejemplo, si una niña sabe que para autoevaluarse favorablemente en el Hacer se esforzará por utilizar y poner en práctica los conocimientos que va aprendiendo en el desarrollo de las clases, 
entonces fortalecerá sus hábitos que contribuyan a su formación integral.

La o el estudiante, al evaluarse a sí mismo, asume la responsabilidad de otorgarse una valoración tanto cualitativa como cuantitativa tomando como referencia central los criterios de evaluación del objetivo holístico del bimestre respectivo. De este modo, la autoevaluación refleja la autopercepción y valoración de la o el estudiante en el transcurso del bimestre.

\section{Relación de los componentes Estudiantes - profesores}

Esta relación es de tipo dialéctica ya que dentro de la enseñanza y aprendizaje, los profesores y los estudiantes llegan a complementar e interactuar diferentes saberes y conocimientos en el aula, es sin duda el medio fundamental donde el profesor despliega sus recursos personales y didácticos para cumplir con su labor que tiene como eje medular la relación con los estudiantes, la relación entre el profesor y el estudiante permite consolidar una adecuada relación armónica "vivir bien" (Huanacuni, 2010) en el aula, como también ambos pueden complementar sus experiencias educativas interactuando e interrelacionándose en cada actividad que puede haber en el curso.

\section{Estudiantes - procesos Autoevaluativos}

La relación estudiantes y procesos Autoevaluativos es de tipo causal ya que el proceso genera la autovaloración propia de cada uno de los estudiantes, por lo que la autoevaluación puede y debe ser un instrumento que facilite atender, respetar $\mathrm{y}$ valorar los distintos ritmos de aprendizaje según las características de los estudiantes; caracterizas relativas como capacidades, estilos de aprendizaje, estrategias cognitivas experiencias y conocimientos previos, motivación, atención, ajuste emocional y social etc.

La autoevaluación es la estrategia por el excelencia para educar con responsabilidad y para aprender a valorar, criticar y reflexionar sobre el proceso de enseñanza y aprendizaje realizado por el profesor, es uno de los medios para que el estudiante conozca y tome conciencia de cuál es su progreso individual en sus procesos formativos.

Este proceso forma parte de otras formas de evaluación para atender a la diversidad, por lo que es necesario utilizar diferentes instrumentos evaluativos para tratar de valorar la progresión de las capacidades habilidades y actitudes de cada estudiante.

\section{Profesores - procesos de Autoevaluación}

Esta relación es de tipo causal, porque de acuerdo a la intervención pedagógica realizada por el profesor se constata el resultado que genera el proceso de la autoevaluación en el desempeño de los estudiantes, como también la autoevaluación debe ser promovida por el profesor como un proceso reflexivo para los estudiantes, sobre las metas; permite contextualizar y analizar el camino recorrido, es decir, a una "comprensión histórica" de la situación y una "comprensión epistemológica y ética" como constituyentes de esa práctica: significa trabajar sobre ella, reflexionar; analizar las posibilidades, los obstáculos, los problemas.

La autoevaluación es la práctica del maestro (as) es un proceso en el que los profesores formulan opiniones valorativas sobre la educación y efectividad de su trabajo en un grupo de estudiantes, este conjunto de valoraciones acerca de propia actividad en el aula, esto significa, entonces, poder objetivar el trabajo por un lado y reconocer la responsabilidad y compromiso. 
El proceso de autoevaluación supone que un profesor, individualmente o con otros compañeros llevan a cabo un auto-examen de su labor como profesor en distintas dimensiones empleando diferentes métodos. Como, los cuestionarios que es uno de ellos.

\section{Concreción del modelo teórico}

Una de las propuestas novedosas dentro del campo de saberes y conocimientos es el referido a la autoevaluación. Al respecto, los documentos oficiales en el cual indica que la Autoevaluación se caracteriza en un instrumento que refleja el desarrollo de las cuatro dimensiones. Este instrumento es el "Cuaderno de Autoevaluación del Estudiante", es único para todas las áreas de saberes y conocimientos, ya que justamente busca la integralidad de la formación y el estudiante, situando su desarrollo en la cotidianidad y su contexto sociocultural.

\section{Procedimientos}

A continuación se presenta, las planificaciones detalladas de cada una de las estrategias de la concreción del modelo, tomando en cuenta los aspectos siguientes: Objetivo holístico, Contenidos, Momentos metodológicos, técnicas, Recursos, Tiempo y Responsabilidades.

\section{Estrategias metodológicas educativas}

\section{Estrategia № 1:}

\section{"Evaluar mi pasado y su influencia en mis comportamientos actuales"}

Esta estrategia enfatiza en la DIMENSIÓN DEL SER principalmente en revisar las influencias en la historia de vida pasada. Para esto cada estudiante construye su historia de vida, identificando dos procesos: lo que más le gusta recordar y lo que no le gusta recordar.
Para proceder:

- Se entrega a cada estudiante una hoja en blanco y un lápiz

- Cada estudiante tiene 20 minutos para escribir su historia de vida enfatizando en sus recuerdos positivos y recuerdos negativos

- Posteriormente en un círculo comparten lo más central de su historia de vida.

- El investigador cierra la actividad valorando la confianza demostrada por los estudiantes al compartir sus historias particulares remarcando que se desarrolló en la dimensión del ser.

\section{Estrategia № 2:}

\section{“Revisar mis deudas emocionales y encontrar la mejor forma de saldarlas"}

Esta estrategia también se enfoca en el desarrollo del SER a partir de una revisión de las deudas emocionales es decir de aquellos procesos personales insatisfechos y que requieren abordarse a través de alternativas que ayuden a saldar esta deuda emocional Para proceder:

- cada estudiante recibe tarjetas para escribir en ellas las emociones que en su vida no han sido suficientemente o no han sido atendidas

- luego recibe otra tarjeta en la que anotan las ideas para resolver esas emociones desatendidas

- en plenaria el investigador reflexiona sobre la importancia de encarar proactivamente las deuda emocionales puesto que estas, como uno no quiera así esto influye en el ser. 


\section{Estrategia № 3}

\section{"Examinar los sentimientos más frecuentes y la forma cómo este afecta mi conducta personal"}

Esta estrategia enfatiza en el desarrollo de la DIMENSIÓN DEL HACER y consiste en revisar con bastante detalle los sentimientos más frecuentes en la vida de los estudiantes y los efectos que tiene en sus conductas

Para proceder

- Se promueve una situación simulada en el sentido de que cada uno de los estudiantes tienen un amigo extraterrestre a quien tiene el deseo de contarle a través de una carta sobre sus sentimientos y la influencia en su conducta

- Una vez que termina de escribir la carta el investigador invita voluntariamente a leer extractos de la misma

\section{Al cerrar la actividad el maestro valora la importancia de los sentimientos en la conducta de los estudiantes}

\section{Estrategia № 4}

\section{"Reconozco cómo soy como persona"}

Esta estrategia se enfoca en el nivel de conocimiento que tiene el estudiante de sí mismo por lo tanto desarrolla la DIMENSIÓN DEL SABER

Para proceder:

- Cada estudiante trabaja su autoconocimiento atreves de la identificación de sus cualidades fortalezas y aspectos a mejorar a si mismo trabajo el auto concepto es decir las creencia que tiene de sí mismo

- Para esto se recure nuevamente a las tarjetas un color para el auto- conocimiento y otro para el autoconcepto

- En plenaria ubicados en círculo comparten con el grupo partes del auto- conocimiento y del autoconcepto

\section{Estrategia № 5}

"Reconstruir mi autoestima y generar una proyección de vida más saludable"

Esta estrategia apunta a fortalecer el desarrollo de la DIMENSIÓN DEL DECIDIR a través de una actitud positiva para reconstruir su autoestima y proyectar iniciativas de vida más saludable.

Para proceder

- Cada estudiante en una hoja en blanco identifica sus principales necesidades y como frente a ellas desarrolla el respeto a los demás

- El investigador genera un ambiente de confianza como para que cada estudiante se sienta motivado a explorar su persona pero a la vez compartir con el grupo.

- Si las condiciones están favorables se pide que de manera voluntaria compartan con el grupo parte de su trabajo.

\section{Implementación de las estrategias}

Es importante considerar tres pasos para la implementación de las estrategias:

\section{Paso 1: Coordinación previa}

Para asegurar que la implementación de las estrategias tengan las mejores condiciones posibles, un primer paso, necesario a trabajar es la coordinación.

Coordinación entre el investigador, y los profesores del campo vida tierra y territorio, y la dirección. La misma que consiste en lo siguiente: 
- Definir día y hora para la realización de las actividades de autoevaluación.

- Definir el lugar donde se desarrollarán las actividades de autoevaluación.

- Establecer las responsabilidades del maestro guía, investigador y estudiantes.

\section{Paso 2: Ejecución de la actividad}

Este paso consiste, propiamente, en el desarrollo de las actividades de autoevaluación, por lo tanto en el trabajo directo con los estudiantes. Su implementación se realizará según los procedimientos definidos para cada estrategia.
Paso 3: Reporte de resultados de la actividad

Una vez realizado cada actividad de autoevaluación, el investigador elaborará un reporte técnico sobre los resultados de la misma así como del procedimiento. Esto con el fin de recuperar experiencias que ayuden a mejorar la investigación.

\section{Evaluación general de la aplicación de las estrategias}

La evaluación se enfocará en la valoración del aporte de las estrategias a fortalecer las dimensiones del desarrollo de la persona. 
Criterios de autoevaluación

\begin{tabular}{|c|c|c|c|c|c|}
\hline Dimensiones & Estrategias & Actividades & $\begin{array}{c}\text { Lo positivo de } \\
\text { las actividades } \\
\text { para mejorar } \\
\text { mi persona }\end{array}$ & $\begin{array}{c}\text { Mis } \\
\text { observaciones } \\
\text { a las } \\
\text { actividades }\end{array}$ & $\begin{array}{r}\text { Sugiero para } \\
\text { mejorar las } \\
\text { actividades }\end{array}$ \\
\hline \multirow[b]{2}{*}{ SER } & $\begin{array}{l}\text { Evaluar mi } \\
\text { pasado y su } \\
\text { influencia en mis } \\
\text { comportamientos } \\
\text { actuales. }\end{array}$ & $\begin{array}{l}\text { "Construyendo mi } \\
\text { historia de vida" }\end{array}$ & & & \\
\hline & $\begin{array}{l}\text { Revisar mis } \\
\text { deudas } \\
\text { emocionales y } \\
\text { encontrar la } \\
\text { mejor forma de } \\
\text { saldarlas. }\end{array}$ & $\begin{array}{l}\text { "El bosque de mis } \\
\text { emociones" }\end{array}$ & & & \\
\hline HACER & $\begin{array}{l}\text { Examinar los } \\
\text { sentimientos más } \\
\text { frecuentes y la } \\
\text { forma cómo estos } \\
\text { afectan mi } \\
\text { conducta } \\
\text { personal. }\end{array}$ & $\begin{array}{l}\text { "Carta a un } \\
\text { extraterrestre" }\end{array}$ & & & \\
\hline SABER & $\begin{array}{l}\text { Reconozco cómo } \\
\text { soy como } \\
\text { persona. }\end{array}$ & $\begin{array}{l}\text { "Mis cualidades, } \\
\text { fortalezas y } \\
\text { Aspectos por } \\
\text { mejorar" } \\
\text { (Autoconocimiento) } \\
\text { "Mis creencias } \\
\text { Acerca de mí } \\
\text { mismo" (auto } \\
\text { concepto) }\end{array}$ & & & \\
\hline DECIDIR & $\begin{array}{l}\text { Reconstruir mi } \\
\text { Autoestima y } \\
\text { Generar una } \\
\text { proyección de } \\
\text { vida más } \\
\text { saludable. }\end{array}$ & $\begin{array}{l}\text { "Me respeto a mí } \\
\text { mismo y por eso } \\
\text { decido ser cada día } \\
\text { mejor persona" }\end{array}$ & & & \\
\hline
\end{tabular}


Resultado de la validación empírica de las estrategias metodológicas para el fortalecimiento de los procesos en la autoevaluación Rigurosidad

Para la tabulación de datos, de las preguntas de test se utilizó el programa IBM SPSS Statistics 22 para analizar e interpretar, la información obtenida, dirigidos a los estudiantes de cada uno de las estrategias metodológicas para el fortalecimiento de los procesos de Autoevaluación.

\section{Consistencia y confiabilidad}

Los datos estadísticos y los resultados obtenidos son confiables ya que tienen las preguntas de test, y tienen una relación con la unidad educativa en Donde se aplicó la propuesta y tiene importancia ya que los profesores del campo Vida Tierra y Territorio desconocían que las estrategias metodológicas ayudaban mucho a los estudiantes en la implementación de la autoevaluación y de la autovaloración de sí mismos en sus procesos formativos.

\section{Resultados: obtenidos del test dirigido} a los estudiantes de cuarto de Secundaria "A" de la Unidad Educativa Libertad de las Américas “ $A$

Considerando los resultados se realizó el respectivo análisis e interpretación que permitió una mejor comprensión de los por menores y otros detalles más que se han suscitado en la ejecución del trabajo.

\section{La aplicación de la estrategia en la} planificación "Evaluar mi pasado y su influencia en mis comportamientos actuales"

Esta estrategia enfatiza en la DIMENSIÓN DEL SER principalmente en revisar las influencias que en la historia de vida pasada de los estudiantes.

\section{CONCLUSIONES}

Una vez finalizado el estudio se obtuvo lo siguiente:

Permitió identificar la existencia de procesos educativos tradicionales y rutinarios de los profesores del Campo Vida Tierra y Territorio de cuarto de secundaria "A". Como también en la Unidad Educativa Libertad de las Américas tienen el conocimiento de la autoevaluación de acuerdo de la ley 070 Avelino Siñani-Elizardo Pérez. Los cuales no son aprovechados adecuadamente en la formación de los estudiantes. Debido al desconocimiento de Metodológicas Educativas que guíen y orienten el uso de las estrategias metodológicas en la autoevaluación en las prácticas educativas, (profesores - estudiantes) por otra parte estos aspectos permitieron establecer el diseño teórico y metodológico desde una perspectiva amplia favorable durante el proceso investigativo

El planteamiento de las estrategias metodológicas como un instrumento de apoyo fomenta a la participación e interacción entre los estudiantes y los profesores del Campo Vida Tierra y Territorio. El proceso genera el intercambio de ideas, pensamientos para abrir espacios de comunicación interacción donde los estudiantes pueden desenvolverse con normal facilidad sin el temor de tener miedo. Gran parte de estos aspectos se consolidan desde el planteamiento secuencial de los 5 estrategias metodológicas educativas como también se elaboran para cada estrategia una planificación de 1, 2, 3, 4,5 los mismos contienen procedimientos y actividades educativas que lo realizan los estudiantes bajo el acompañamiento del profesor. 
El estudio fue validado por preguntas de test las cuales se realizaron un antes y después de cada estrategia lo cual ayudó a conocer y valorar las aptitudes, capacidades, potencialidades, amor y concentración del estado de los estudiantes en su personalidad. Las estrategias resultan ser viables como los recursos, las actividades los contenidos, los procedimiento, por ser novedosos en el labor de los estudiantes y de los profesores/as del Campo Vida Tierra y Territorio, ya que logra convertirse en un instrumentó didáctico para lograr grandes resultados con los profesores, siempre pensando en el beneficio de ellos y aquellas personas a quienes van dirigir su actuar profesional con el uso de las estrategias metodológicas le ayude adecuadamente con el objetivo de mejorar la autovaloración.

El producto obtenido de cada estrategia conlleva a la expresión propia de la capacidad analítica, crítica, reflexiva, y sobre todo la autoconciencia, el autoconocimiento y la autovaloración de cada uno de los estudiantes en el aula.

\section{REFERENCIAS}

Díaz Barriga Arceo, F. (2003). Cognición situada y estrategias para el aprendizaje significativo. Revista electrónica de investigación educativa, 5(2), 1-13

González J. (2011). Desarrollo sociológico (página de google+) p.1 Recuperado de: https://javierprietogonzalez.blogspot.com/ 2011/01/emilio- durkheim-resumen.html

Hernández Sampieri, R., Fernández Collado, C., \& Baptista Lucio, P. (2010). Metodología de la investigación

Hernández, R., Fernández, y Baptista, p. (2006). Metodología de la investigación, 4. A edición México, Mc Graw Hill

Huanacuni Mamani F. (2010) Vivir Bien Buen Vivir Junio impresión. cdr - ESCR- Net pp.12- 13

Ministerio de Educación (2014). Unidad de Formación Nro. 15 "Estrategias
Metodológicas en la Educación de Personas Jóvenes y Adultas". Cuadernos de Formación Continua. Equipo PROFOCOM. La Paz p. 11

Ley de la Educación № 070 "Avelino Siñani Elizardo Pérez" PAÍS Bolivia 10/06/2012 Documento compartido por el SIPI PUBLICACIÓN ORIGINAL Gaceta Oficial, Artículo 3. P, 4 A. 4, p, 6. Artículo 6. P, 7,8

Ley de la Educación № 070 "Avelino Siñani Elizardo Pérez" país Bolivia fecha de 10/06/2012 Documento compartido por el SIPI PUBLICACIÓN ORIGINAL Gaceta Oficial, 1 (páginas de google +). Recuperado de www.gacetaoficialdebolivia.gob.bo

Pérez G., (2013). Estrategias metodológicas y técnicas para la investigación social. p. 5

Palacios (1978). Módulo Fundamentos del Desarrollo De la Tecnología Educativa (Bases-Psicopedagógicas) p. 10

Rodríguez, ME. (2011). "Avelino Siñani Elizardo Pérez" y el Proyecto Educativo p.1 (Páginas de Google+) recuperado de http://www.lapatriaenlinea.com/index.php /somos-noticias.html\%3Ft\%3Del- dia-de-lamujer-boliviana\%26nota\%3D44370?t=leyavelino-sinani-elizardo-perez-y-el-proyectoeducativo\&nota $=69185$

Roncal Vattuone, X. (2014). En la búsqueda de la calidad universitaria Bolivia, Colombia, Cuba y México. Revista Integra Educativa, [vivir bien] p, 7(2), 137-158. (Páginas de google+) Recuperado de http://www.scielo.org.bo/scielo.php?scri pt=sci_arttext\&pid=S1997404320140 00200009\&lng=es\&tlng=es.

Ronda pupo Guillermo Armando. (2002, Marzo 11. un concepto). Un concepto de Estrategia. P. 1 (páginas de google+) recuperado de https://www.gestiopolis.com/unconceptode-estrategia/ 